\title{
Comunicação
}

[Communication]

\section{Atividade in vitro do óleo essencial de Origanum vulgare frente à Sporothrix Schenckii}

[In vitro activity of the essential oil of Origanum vulgare against Sporothrix schenckii]

\author{
M.B. Cleff ${ }^{\text {, A.R.M. Meinerz }}{ }^{1}$, L.F.D. Schuch ${ }^{1}$, M.R.A. Rodrigues ${ }^{2}$, M.C.A. Meireles ${ }^{2}$, J.R.B. Mello ${ }^{1}$ \\ ${ }^{1}$ Aluno de pós-graduação - UFRGS - Porto Alegre, RS \\ ${ }^{2}$ Universidade Federal de Pelotas - Pelotas, RS
}

A esporotricose, micose subcutânea causada pelo fungo geofílico Sporothrix schenckii, apresenta distribuição mundial, principalmente nas regiões tropicais e subtropicais, acomete o homem e várias espécies de animais. A espécie felina está freqüentemente envolvida nos relatos zoonóticos, em que a transmissão do agente ao homem ocorre por meio de arranhaduras, mordeduras ou por contato direto com as lesões de felinos com esporotricose. Estudos sugerem que a transmissão é facilitada pela grande quantidade de leveduras nas lesões desses animais, assim como a presença de microrganismos nas unhas e na cavidade bucal (Barros et al., 2004; Souza et al., 2006).

Em relação ao tratamento da esporotricose, os iodetos foram as primeiras substâncias utilizadas para as formas cutâneas e linfocutâneas da micose (Schubach et al., 2004; Catalán e Montejo, 2006). Na década de noventa, o itraconazol, antifúngico do grupo dos triazóis, emergiu como alternativa terapêutica eficaz e com reduzidos efeitos adversos e foi considerado o tratamento de eleição para felinos (Catalán e Montejo, 2006). No entanto, alguns autores têm relatado a ocorrência de falhas terapêuticas com o uso do itraconazol para tratamento de felinos com esporotricose (Schubach et al., 2004).

A pesquisa de substâncias ativas oriundas das plantas tem despertado interesse, em função da grande diversidade de compostos com possível ação antimicrobiana. Dentre as diversas espécies avaliadas quanto à ação terapêutica, destaca-se o

Recebido em 24 de maio de 2007

Aceito em 1 de fevereiro de 2008

Endereço para correspondência (corresponding address)

Rua Xavier Ferreira, 618 - 96010-540 - Pelotas, RS

E-mail: emebrum@bol.com.br
Origanum vulgare, que é amplamente utilizado na culinária e na medicina popular. O seu óleo essencial apresenta alta estabilidade, ausência de contaminação microbiológica e diversidade de componentes químicos, principalmente $\mathrm{o}$ carvacrol e o timol, com atividade antimicrobiana comprovada (Lambert et al., 2001; Baydar et al., 2004).

Este estudo teve como objetivo avaliar a atividade in vitro do óleo essencial de Origanum vulgare frente a Sporothrix schenckii.

Amostras de orégano comercial (O. vulgare) de origem chilena foram submetidas à extração por hidrodestilação em aparelho de Clevenger, segundo a Farmacopéia Brasileira IV (1988), para obtenção do óleo. Após a extração, o óleo foi seco em sulfato de sódio anidro $\left(\mathrm{Na}_{2} \mathrm{SO}_{4}\right)^{1}$, e concentrado sob $\mathrm{N}_{2}^{2}$ ultra puro $(99,9 \%)$, armazenados em frascos âmbar e mantidos sob refrigeração.

O óleo essencial de $O$. vulgare foi analisado em cromatógrafo a gás com detector de ionização de chama (GC/FID) ${ }^{3}$, e caracterizado em função do conteúdo de compostos fenólicos, timol e/ou carvacrol. Para isso, foi preparada uma solução do óleo a $5000 \mathrm{mg} / \mathrm{l}$ em hexano e soluções de 40mg/l dos padrões: $\alpha$-pineno, canfeno, $\beta$ pineno, mirceno, $\alpha$-terpinemo, $\quad p$-cimeno, limoneno, 1,8-cineol, $\gamma$-terpineno, terpinoleno, linalol, 4-terpineol, $\alpha$-terpineol, timol, carvacrol, das quais foram injetadas $1 \mu 1$. Os constituintes foram identificados por comparação entre $o$ tempo de retenção desses padrões e das amostras.

\footnotetext{
${ }^{1}$ Merck - Darmstadt, Germany

${ }^{2}$ White Martins - São Paulo, Brasil.

${ }^{3}$ Schimatzu-17 $7^{\mathrm{A}}$ - Tóquio, Japão.
} 
Para o teste de susceptibilidade ao óleo essencial, foram utilizados sete isolados de S. schenckii. As amostras foram provenientes de esporotricose cutânea de felinos $(n=5)$ e de esporotricose humana $(\mathrm{n}=2)$, estocadas na micoteca do laboratório de micologia da Faculdade de Veterinária da Universidade Federal de Pelotas (UFPel). Os isolados foram mantidos na sua fase miceliana a partir de subculturas em tubos contendo meio Brain Heart Infusion, refrigerados em temperatura média de $4^{\circ} \mathrm{C}$, realizando-se subcultivos com intervalos de seis meses.

A suscetibilidade de $S$. schenckii ao óleo foi avaliada por meio da técnica de microdiluição em caldo, de acordo com o protocolo descrito no documento NCCLSM27-A2 (Reference..., 1997), que descreve a técnica de suscetibilidade antifúngica frente aos fungos leveduriformes adaptada para um fitofármaco.

O óleo foi submetido a uma série de nove diluições em logaritmo de base 2, no meio sintético RPMI $1640^{4}$, acrescido do tampão de ácido morfolino propanosulfônico, obtendo-se óleo em concentrações de 2,0 até $0,004 \%$. Essas concentrações foram distribuídas no sentido das colunas das microplacas em volumes de $100 \mu \mathrm{l}$.

Os inóculos fúngicos, referentes aos isolados testados, foram preparados a partir de colônias jovens (48-72h) da fase leveduriforme de $S$. schenckii, as quais foram ressuspensas em tubos contendo solução salina estéril, homogeneizadas em agitador e ajustadas à turbidez 0,5 da escala de MacFarland. A suspensão fúngica foi diluída em solução salina (1:50), homogeneizada, e submetida à diluição em meio RPMI 1640 (1:20). O inóculo foi distribuído no sentido das linhas das microplacas em volumes de $100 \mu \mathrm{l}$.

Os testes de suscetibilidade ao óleo foram realizados em duplicata, sendo uma coluna da microplaca utilizada para o controle positivo (RPMI 1640 e inóculo fúngico) e outra coluna para o controle negativo (RPMI 1640). As microplacas foram incubadas a $35^{\circ} \mathrm{C}$ sob agitação branda $(40$ ciclos/min) por até cinco dias, quando foi realizada a leitura da concentração inibitória mínima (CIM). A CIM foi obtida pela comparação visual do crescimento do agente ocorrido nos poços referentes às diferentes concentrações testadas, com o seu crescimento no controle positivo. A menor concentração capaz de produzir proeminente inibição do crescimento da levedura em relação ao controle-positivo foi identificada como a CIM do óleo essencial para S. schenckii.

A CIM do óleo essencial do orégano para $S$. schenckii foi de $0,25 \%(250 \mu \mathrm{l} / \mathrm{ml})$, para todos os isolados testados. Outros estudos comprovaram a atividade in vitro do óleo essencial de $O$. vulgare e seus componentes químicos frente a várias espécies de fungos filamentosos como Aspergillus niger, $A$. flavus, A. ochraceus, A. parasiticus, Penicilium italicum e Fusarium oxysporum (Buchanan e Shepherd, 1981; Paster et al., 1990; TantaouiElaraki et al., 1993; Daouk et al., 1995), assim como frente a espécies de Candida e Cryptococcus neoformans (Manohar et al., 2001; Chami et al., 2004). Não foi, todavia, avaliada a sua atividade frente ao $S$. schenckii.

A homogeneidade observada para os valores da CIM foi diferente do que se observa em avaliação de substâncias antifúngicas puras, nas quais se verifica grande amplitude entre os valores da CIM. Esse fato explica-se, provavelmente, pela diversificada composição química do óleo essencial, que dificulta a utilização pelos microrganismos de rotas metabólicas alternativas que lhe confiram maior ou menor resistência, como ocorre nos casos de princípios ativos isolados (Rios et al., 1988).

Em relação aos compostos detectados pela análise cromatográfica da amostra de orégano utilizada, observou-se maior concentração de timol em relação ao carvacrol (Fig.1). Mesmo sendo reconhecidas as propriedades antimicrobianas do óleo essencial de Origanum spp, ainda não foi possível determinar com clareza o mecanismo de ação que justifique essa atividade. Acredita-se que a ação antimicrobiana possa ser decorrente da alteração de diversas enzimas, incluindo aquelas envolvidas com a produção de energia e a síntese de componentes estruturais. Em geral, o estudo do mecanismo de ação dos óleos essenciais tem usado metodologia comum na tentativa de ilustrar os efeitos prejudiciais que ocorrem na membrana celular (Lambert et al., 2001).

O óleo essencial de $O$. vulgaris demonstrou atividade in vitro frente a $S$. schenckii, utilizando a técnica de microdiluição em caldo adaptada a um fitofármaco, o que justifica novas análises com o óleo do orégano, incluindo estudos in vivo.

Palavras-chave: Sporothrix schenckii, Origanum vulgare, orégano, tratamento

${ }^{4}$ RPMI 1640 medium - Sigma-Aldrich - St. Louis, USA. 


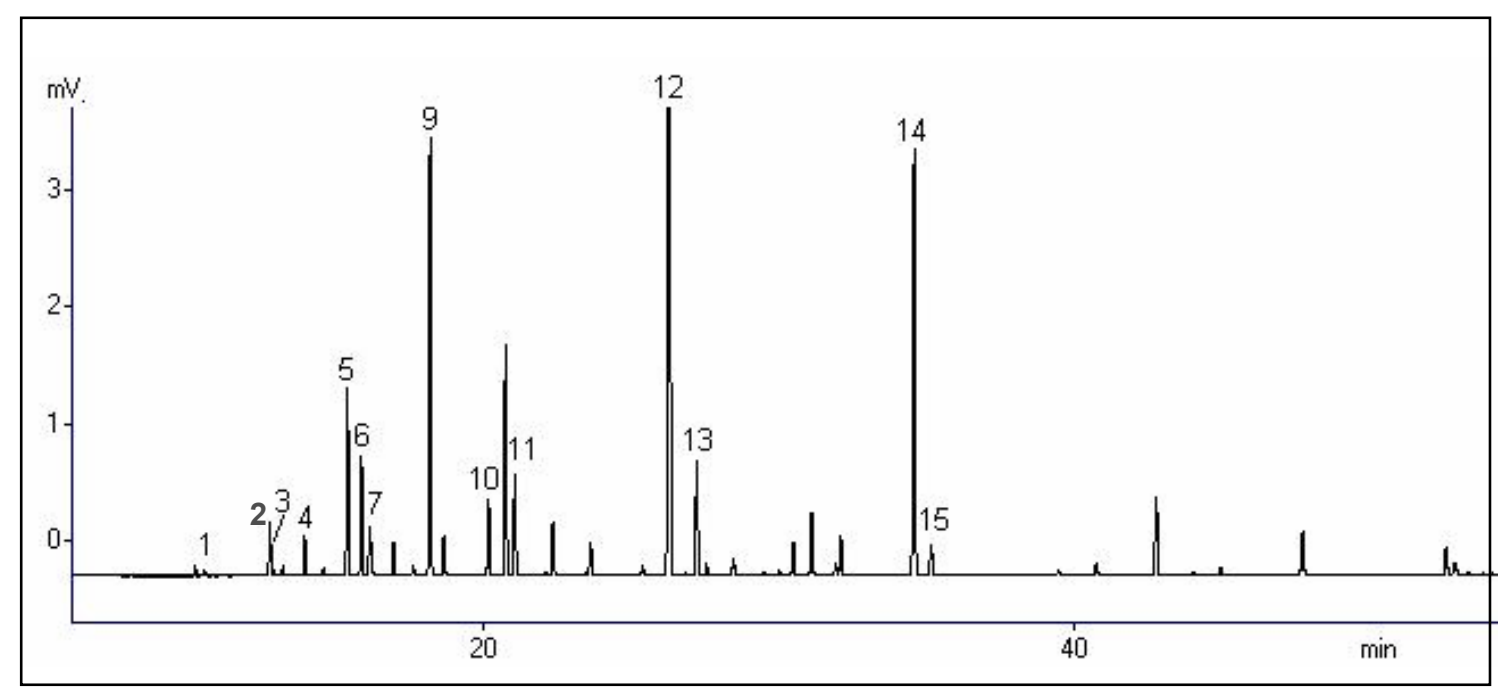

Figura 1. Cromatograma - óleo essencial de orégano, solução de 5000mg/l em hexano. Picos dos padrões: 1) $\alpha$-pineno; 2) canfeno; 3) $\beta$-pineno; 4) mirceno; 5) $\alpha$-terpineno; 6) $p$-cimeno; 7) limoneno; 8) 1,8 cineol; 9) $\gamma$-terpineno; 10) terpinoleno; 11) linalol; 12) 4-terpineol; 13) $\alpha$-terpineol; 14) timol; 15) carvacrol.

\begin{abstract}
In vitro activity of the essential oil Origanum vulgare against Sporothrix schenckii was determined by the MIC (Minimum Inhibitory Concentration). For this, seven samples of S. schenckii were studied, two isolated from two cases of human sporotrichosis and five isolated from cats. Analysis of the essential oil was carried out in a gas chromatograph (GC/FID) for the identification and quantification of thymol and carvacrol (antifungal agents). MIC was obtained based on the microdilution method according to the adapted document NCCLS-M 27A2 for fitopharmacy. All the isolates presented sensibility to the essential oil. S. schenckii was inhibited in a concentration of $0.25 \%(250 \mu \mathrm{l} / \mathrm{ml})$. Chromatographic analysis showed that thymol concentration was bigger than carvacrol. The antifungal activity demonstrated by the essential oil of $\mathrm{O}$. vulgare against $\mathrm{S}$. Schenckii stimulates the accomplishment of more studies, including in vivo studies.
\end{abstract}

Keywords: Sporothrix schenckii, Origanum vulgare, oregano, treatment

\section{REFERÊNCIAS BIBLIOGRÁFICAS}

BARROS, M.B.L.; SCHUBACH, A.O.; DO VALLE, A.C.F. et al. Cat-transmitted sporotrichosis epidemic in Rio de Janeiro, Brazil: Description of a series of cases. Clin. Infect. Dis. Soc. Am., v.38, p.529-535, 2004.

BAYDAR, H.; SAGDIÇ, O.; OZKAN, G. Antibacterial activity and composition of essential oils from Origanum, Thymbra and Satureja species with commercial importance in Turkey. Food Control, v.15, p.169-172, 2004.
BUCHANAN, R. L.; SHEPHERD, A. J. Inibition of Aspergillus parasiticus by timol. $J$. Food Sci., v.46, p.976-977, 1981.

CATALÁN, M.; MONTEJO, J.C. Antifúngicos sistémicos. Rev Iberoam Micol., v.23, p.39-49, 2006.

CHAMI, F.; CHAMI, N.; BENNIS, S. et al. Evaluation of carvacrol and eugenol as prophylaxis and treatment of vaginal candidiasis in an immunosuppressd rat model. J. Antimicrob. Chemother., v.54, p.909-914, 2004. 
DAOUK, R.K.; DAGHER, S.; SATTOUT, E. Antifungal activity of essential oil of Origanum syriacum L. J. Food Prot., v.58, p.1147-1149, 1995.

LAMBERT, R.J.W.; SKANDAMIS, P.N.; COOTE, P.J. A Study of the minimum inhibitory concentration and mode of action of oregano essencial oil, thymol and carvacrol. J. Applied Microbiol., v.91, p.453-462, 2001.

MANOHAR, V; INGRAM, C.; GRAY, J. et al. Antifungal activities of Origanum oil against Candida albicans. Mol. Cel. Biochem., v.228, p.111-117, 2001.

PASTER, N.; JUVENT, B.J.; SHAAYA, E. et al. Inhibitory effect of oregano and thyme essential oils on mould and foodborne bacteria. Lett. Applied Microbiol., v.11, p.33-37, 1990.

REFERENCE method for broth dilution antifungal susceptibility testing of yeast.
Approved standars 27. Villanova, Pa: NCCLS, 1997.

RIOS, J.L.; RECIO, M.C.; VILLAR, A. Screening methods for natural products with antimicrobial activity: A review of the literature. J. Athnopharmacol., v.23, p.127-149, 1988.

SCHUBACH T.M.P.; SCHUBACH, A.O.; KAMOTO BARROS, T.M.B.L. et al. Evaluation of an epidemic of sporotrichosis in cats: 347 cases (1998-2001). J. Am. Vet. Med. Assoc., v.224, p.1623-1629, 2004.

SOUZA, L.L.; NASCENTE, P.S.; NOBRE, M.O. et al. Isolation of Sporothrix schenckii from the nails of healthy cats. Braz. J. Microbiol., v.37, p.303-305, 2006.

TANTAOUI-ELARAKI, A.; FERHOUT, H.; ERRIFI, A. Inhibition of the fungal asexual reproduction stages by three Moroccan essential oils. J. Essen. Oils Res., v.5, p.535-543, 1993. 